\title{
DESIGN OF A MERGING BEAMLET PRE-ACCELERATOR FOR AN ELECTROSTATIC QUADRUPOLE ACCELERATOR (ESQ)
}

\author{
C. F. Chan and M. C. Vella \\ Lawrence Berkeley Laboratory, Berkeley CA 94720
}

\begin{abstract}
An experiment is planned to merge $200 \mathrm{~mA}$ of multibeamlet $\mathrm{H}^{-}$at $100 \mathrm{keV}$ into a single channel electrostatic quadrupole accelerator (ESQ), which will boost the beam to $200 \mathrm{kV}$. The experiment is a collaboration based on a Japan Atomic Energy Research Institute (JAERI) volume $\mathrm{H}^{-}$plasma source and focused multi-aperture extractor, mated to the LBL $200 \mathrm{kV}$ prototype ESQ. A 2D particle code (WOLF) has been used to design a merging beamlet pre-accelerator. The key merging elements are a focus ring to enhance the focusing of the extractor and a gradient grid to produce a merged beam profile which matches ESQ acceptance and minimizes emittance growth.
\end{abstract}

\section{INTRODUCTION}

This paper presents a design for an experiment to demonstrate merging of the beamlets from a multi-aperture extractor into a prototype ESQ. The experiment will use a JAERI volume generation $\mathrm{H}^{-}$plasma source and focused extractor [1], mated to the LBL $200 \mathrm{kV}, 200 \mathrm{~mA} \mathrm{ESQ}$ prototype [2]. If successful, this combination can be scaled to amperes of megavolt negative ion based neutral beams, for heating and current drive in future tokamak such as the International Thermonuclear Experimental Reactor (ITER).

Two types of negative ion accelerators are under development. Conventional electrostatic (ES) Pierce type dc accelerators have the advantage of being proven with low currents up to several megavolts. Also, they directly match the capabilities of available $\mathrm{H}^{-} / \mathrm{D}^{-}$cesiated volume plasma sources. The reactor relevant challenges for ES accelerators are voltage holding and insulator lifetime. Electrons stripped from the negative ion beam by gas, plus secondaries, can generate a high $\mathrm{x}$-ray flux. The LBL group is developing the ESQ accelerator because it is mechanically compatible with long, low (electrical) stress high voltage insulators $(\leq 4$ $\mathrm{kV} / \mathrm{cm}$ ). Also, the quadrupole fields are expected to filter out energy mismatched particles, reducing $x$-ray loads.

The practical challenges for a multi-beamlet merging experiment are: (1) Match the ESQ acceptance requirements; (2) Demonstrate $\mathrm{H}^{-}$current density that scales to ITER requirements; (3) Achieve acceptable emittance growth within the ESQ.

\section{LBL $200 \mathrm{KV}$ ESQ}

Matching requirements for the LBL $200 \mathrm{kV}, 200 \mathrm{~mA}$ (proton equivalent) ESQ are: $100 \mathrm{keV}$ input energy; Input radius $\leq 1 \mathrm{~cm}$; and Convergence $-10 \mathrm{mrad}$ to $-30 \mathrm{mrad}$ for a circular beam. Previous experiments used single channel positive and negative ion beams. The nominal $200 \mathrm{~mA}$ current was demonstrated with a space charge equivalent 100 $\mathrm{mA} \mathrm{He}^{+}$beam [2].

This ESQ has three quadrupoles in the matching section, and two quadrupoles forming a $100 \mathrm{kV}$ acceleration section, as illustrated in Fig.1. The design is modular, and could be expanded in $100 \mathrm{kV}$ sections to over $1 \mathrm{MV}$. The ESQ has a $3.5 \mathrm{~cm}$ diameter aperture. If the input conditions are met, the beam envelope can be kept within $80 \%$ of the aperture diameter, and emittance growth due ESQ electric field nonlinearities should be minimal [3]. The short length of the 200 $\mathrm{kV}$ configuration makes it very tolerant of input emittance.

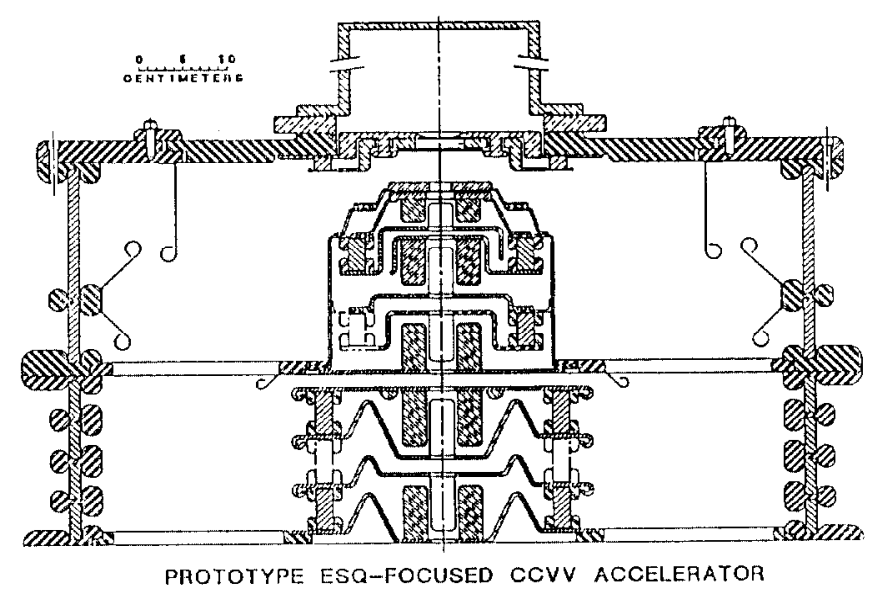

Figure 1. Drawing of the LBL $200 \mathrm{kV}, 200 \mathrm{~mA}$ ESQ. The first three modules are used for matching, and the second two modules for acceleration from $100 \mathrm{keV}$ to $20 \mathrm{keV}$.

\section{JAERI FOCUSED EXTRACTOR}

The JAERI plasma source will be similar to the type tested at JAERI and Cadarache, and planned for JT-60U [3] The usual cesium seeding is planned to increase output and reduce gas pressure. The JAERI focused extractor [1] uses curved plasma and extraction grids, with nineteen apertures and embedded magnets for electron suppression. Three sets of grids were built with nominal geometric focal lengths of 10 $\mathrm{cm}, 15 \mathrm{~cm}$ and $20 \mathrm{~cm}$ (i.e., plasma grid radius of curvature). 
The $15 \mathrm{~cm}$ and $10 \mathrm{~cm}$ grids were tested at JAERI in 1992 [1]. Except for curvature, these are similar to the flat grids used in the large JAERI injectors [4], including embedded magnets to bury electrons in the extraction grid. The beamlets are defined by $0.45 \mathrm{~cm}$ radius holes in the plasma and extraction grids. A side view of the nominal $15 \mathrm{~cm}$ grid set is shown in Fig. $2 \mathrm{a}$, and a flat projection is shown in Fig. $2 b$.

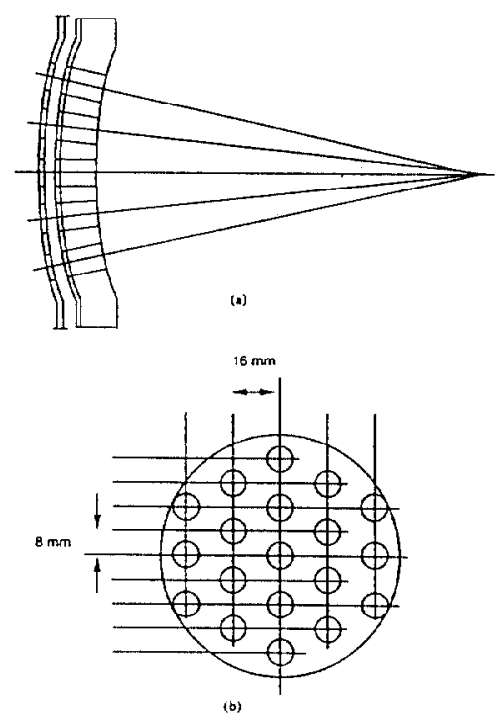

Figure 2. The layout of the JAERI focuscd plasma and extraction grid design is illustrated: (a) Side view, $15 \mathrm{~cm}$ focal length; and (b) Projection of the beamlet layout, with one, six, and twelve beamlets rings.

\section{2D SIMULATION OF BEAM MERGING}

The goal of these studies is to provide a conceptual design for a beamlet merger which would match the JAERI extractor and the LBL ESQ. The merged beam should satisfy the acceptance requirements of the latter as mentioned in Sec. II above. To accomplish this we propose two additional optics elements: (1) focus ring; and (2) gradient grid. A focus ring on the exit side of the extraction grid, just outside the last ring of beamlets, increases the curvature of the potential contours for the outer beamlets. The gradient grid is used to tune the envelope convergence. A relatively long gap to the ESQ is required to get a good input match, which should experience low emittance growth, even in a long acceleration channel.

As mentioned previously, limiting emittance growth due to ESQ non-linearities requires that the envelope radius remain within $\leq 80 \%$ of the nominal aperture. This will be possible if the ESQ acceptance requirements are met, but also requires that the merging be accomplished in a way which minimizes the electrostatic energy of the merged beam. This means we should stack the beamlets against each other with minimal overlap. In the planned experiment, given the $0.45 \mathrm{~cm}$ beamlet radius and the $\leq 1 \mathrm{~cm}$ ESQ acceptance radius, beamlet overlap after merging is unavoidable.

To adapt to the 2D symmetry of WOLF[5,6], the simulated beam geometry was a set of concentric, focused cylindrical ringlets, which were set at radii equivalent to the beamlets from the JAERI extraction grid. The ringlets were launched with the energy expected for the beam at the extraction grid during the LBL experiment, $6.5 \mathrm{keV}$. They were given a Maxwellian distribution equivalent to a temperature of $1 \mathrm{eV}$.

Results for two geometries are presented: (1) Two ringlets, which corresponds to seven apertures with total current $\leq 100 \mathrm{~mA}$; and (2) Three ringlets, which corresponds to using the nineteen available apertures with total current $\leq$ $200 \mathrm{~mA}$. Current in the ringlets was distributed in the same ratio as the number of apertures of the JAERI layout, i.e., $1: 6$, for two ringlets (seven holes), or, 1:6:12, for threc ringlets (nineteen holes). This distribution most closely resembles the current distribution of the beamlet layout, but perhaps introduces some extraneous effects on emittance. For comparison with the experiment, it is convenient to refer to these configurations as "seven holes" and "nineteen holes".

The effect of a gradient grid on convergence of the seven hole trajectories is illustrated in Fig.3a, where a large aperture, $60 \mathrm{kV}$ gradient grid with a thickness of $1 \mathrm{~cm}$ is added $6.0 \mathrm{~cm}$ from the center of the extraction grid and $12.0 \mathrm{~cm}$ from the ESQ entrance. The corresponding phase space is illustrated in Fig. $3 \mathrm{~b}$. Envelope convergence is greatly reduced after the beam pass through the second gap. The $0.9 \mathrm{~cm}$ radius is a good match to the ESQ. A quantitative measure of the collective beam envelope was obtained from WOLF by calculating the rms parameters of the projectional phase space ellipse. These rms parameters were used to calculate the convergence angle of a $\mathrm{K}-\mathrm{V}$ beam with equivalent projectional cmittance. Based on this procedure, the beam in Fig.3a has a $\mathrm{K}-\mathrm{V}$ equivalent envelope convergence of $-24 \mathrm{mrad}$, which matches the acceptance of the $200 \mathrm{~mA}$ ESQ. In general, increasing the gradient grid voltage (with respect to the extractor) increases the beam envelope convergence, with a small change in the envelope radius.
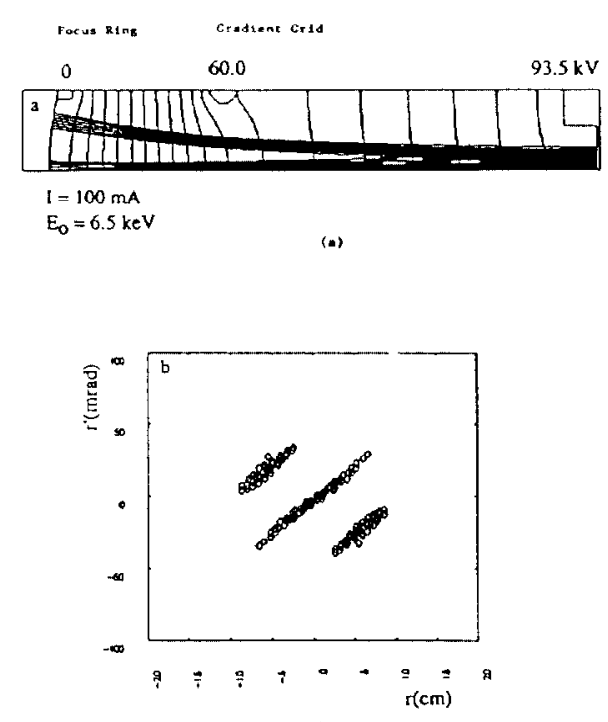

Figure 3. WOLF simulations for a $100 \mathrm{~mA} \mathrm{H}^{-}$beam with a center beam and one ringlet, accelerated from $6.5 \mathrm{kcV}$ to 100 $\mathrm{keV}$. Beam trajectories are plotted in (a) and the $r-r^{\prime}$ phase space is shown in (b). 
Trajectories and phase space diagrams for a nineteen hole (i.e., three ringlet) design are illustrated in Fig. $4 \mathrm{a}$ and $\mathrm{b}$. Changes from the previous design are an increased gap between the extraction grid and gradient grid (to $10 \mathrm{~cm}$ ). Beam radius at the ESQ entrance is about $1 \mathrm{~cm}$ and convergence is acceptable, $-30 \mathrm{mrad}$.
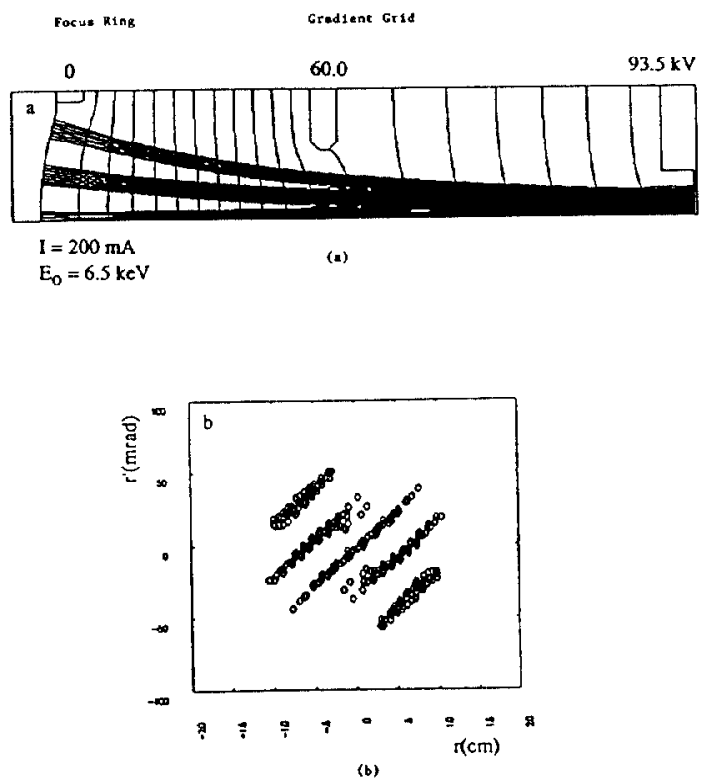

Figure 4. WOLF simulations for a $200 \mathrm{~mA} \mathrm{H}$ beam with a center beam and two ringlets, accelerated from $6.5 \mathrm{keV}$ to 100 $\mathrm{keV}$. Beam trajectories are plotted in (a), and the $r-r$ 'phase space is shown in (b).

The large gap layout illustrated in Fig.4a, with focus ring and gradient grid, has been chosen for the joint experiment between JAERI and LBL. Assuming the plasma grid is masked and the appropriate focus ring installed, this design has been shown computationally to also work well with seven beamlets. WOLF simulations indicate that for seven beamlets, the gradient grid should be at $90 \mathrm{kV}$. With $100 \mathrm{~mA}$ $\mathrm{H}^{-}$, the envelope was $0.65 \mathrm{~cm}$ in radius, with a $-12 \mathrm{mrad}$ convergence. This is an easy match to the $200 \mathrm{kV}$ ESQ.

The long path from the extraction grid to the ESQ in the experimental layout raises concerns about stripping losses. However, progress in volume source technology has significantly reduced operating pressure in recent years. Also, this ESQ was designed with a high conductance to handle gas from older sources, with higher pressure than now expected. Estimates are that stripping losses associated with the longer path length will be a small fraction of the losses within the extractor.

The conceptual design for the nineteen hole merger shown in Fig.4a offers a good match to the acceptance of the $200 \mathrm{kV}$ ESQ, and the expected current should scale to an ITER proof of principal demonstration. The key optics elements are a focus ring at the perimeter of the extraction grid and a large aperture gradient grid. The focus ring effectively increases the curvature of the extraction grid, and the gradient grid insures the flexibility to match the FSQ acceptance. This design is adaptable to also testing seven beamlets. Self-consistent 3D simulations are planned in the future to refine the design and to study emittance growth.

\section{ACKNOWLEDGMENT}

The authors are pleased to acknowledge constructive technical suggestions from W. S. Cooper at LBL, and Y. Okumura and T. Inoue at JAERI. This work was supported by the Director, Office of Energy Research, Office of Fusion Energy, Development and Technology Division, of the U.S. Department of Energy under Contract No. DE-AC03$76 \mathrm{SF} 00098$.

\section{REFERENCES}

[1] T. Inoue, M. Hanada, M. Mizuno, Y. Okumura, Y. Ohara, M. Tanaka, Y. Suzuki, and K. Watanabe, "Development of a Multi-Ampere $\mathrm{H}^{-}$Ion Source at JAERI," Proc. Sixth Intl. Symp. on Production and Neutralization of Negative Ions and Beams, Upton, New York, USA, November 9-13, 1992. (to be published)

[2] J.W. Kwan, G.D. Ackerman, O.A. Anderson, C.F. Chan, W.S. Cooper, G.J. deVries, W. B. Kunkel, K.N. Leung, P. Purgalis, W.F. Steele, and R.P. Wells, Rev. Sci. Instrum. 62 (6), p. 1521 (1991).

[3] I. Haber, "Simulation of Low Emittance Transport", Proc. INS Intl. Symp. on Heavy Ion Accelerator and Their Application to Inertial Fusion, Tokyo, p.451 (1984).

[4] Y. Okumura, "Negative Ion Based Neutral Beam Injector for JY-60U," Proc. Sixth Intl. Symp. on Production and Neutralization of Negative Ions and Beams, Upton, New York, USA, November 9-13, 1992. (to be published)

[5] W. S. Cooper, K. Halback and S. B Magyaryi, Proc. 2nd Symp. on Ion Sources and Formation of Ion Beams, Berkeley, LBL-3399 (1974).

[6] C.F. Chan, W.S. Cooper, J.W. Kwan, and W.F. Steele, Nucl. Inst. and Meth. in Phys. Res. A306, p. 112 (1991). 